\title{
Quark spin properties at high $\mathrm{x}$
}

\author{
Jerrold Franklin* \\ Department of Physics \\ Temple University, Philadelphia, PA 19122-6082
}

(August 6, 2004)

\begin{abstract}
A general three quark bound state satisfying the Pauli principle, and conserving angular momentum and isospin, is used to investigate the spin structure of nucleons at high $x$. It is shown if $F_{1 n} / F_{1 p} \rightarrow \frac{1}{4}$, then both $A_{1 p}$ and $A_{1 n}$, as well as quark spin distribution $\Delta u / u$, will approach 1 as $x \rightarrow 1$ in polarized deep inelastic scattering. The spin distribution $\Delta d / d$ does not approach 1 , but is bound by the limits $-\frac{1}{3} \leq \Delta d / d \leq 0$ for any $x$.
\end{abstract}

*Internet address: Jerry.F@TEMPLE.EDU 
The region of high Bjorken $x$ in deep inelastic scattering (DIS) has become of great interest recently because of the possibility of testing theoretical predictions for the asymmetry parameters $A_{1 p}(x)$ and $A_{1 n}(x)$ as $x \rightarrow 1$. A recent experiment has measured $A_{1 n}$ up to $x=0.6$ [1], and planned experiments may be able to measure the asymmetries at higher $\mathrm{x}$. Also, there is a renewed recognition that the absence of sea and explicit gluon effects for $x$ above 0.3 makes the high $x$ region a good testing ground for valence quark theories of DIS. In this note, we examine the general properties of nucleon quarks ( $u$ and $d$ ) obeying the Pauli principle in a general three quark bound state, and show that there are strong model independent constraints relating the behavior of unpolarized and polarized DIS at high $x$.

We start by constructing the most general spin $\frac{1}{2}$, isospin $\frac{1}{2}$ bound state of three nucleon quarks. For a spin up proton, this can be written as $^{1}$

$$
\psi(\text { uud })=N \operatorname{uud}\{\uparrow \uparrow \downarrow[\phi(123)+\phi(213)]-\uparrow \downarrow \uparrow \phi(123)-\downarrow \uparrow \uparrow \phi(213)\} .
$$

This general proton wave function depends on a single three-body spatial function $\phi(123)$. The three quark coordinates could be the position, the momentum or, to apply directly to DIS, the Bjorken $x$ of each quark. The normalization $N$ is model dependent because of interference between $\phi(123)$ and $\phi(213)$. The state in Eq. (1) is to be considered as the uud part of a completely symmetrized wave function of the form

$$
\Psi=\frac{1}{\sqrt{3}}[\psi(\text { uud })+\psi(\mathrm{udu})+\psi(\mathrm{duu})] .
$$

In this symmetrization, the state $\psi(\mathrm{udu})$ is obtained from $\psi(\mathrm{uud})$ by interchanging the second and third spin and space coordinates as well as the indicated quark type, with a similar interchange of the first and third coordinates for $\psi(\mathrm{duu})$. For most purposes, including calculating structure functions for DIS, it is sufficient to use only the uud order $[2]{ }^{2}$ Using all three quark orders would just involve doing the same calculation three times.

We arrived at the proton wave function in Eq. (1) by the following steps:

1. We assume that the quarks are antisymmetric in the color degree of freedom, so that the Pauli principle for the two u quarks requires the spatial and spin functions to appear in the combination $[\uparrow \downarrow \uparrow \phi(123)+\downarrow \uparrow \uparrow \phi(213)]$ in the last two terms of Eq. (1).

2. For the three quarks to be in a $J=\frac{1}{2}$ spin state, application of the step-up operator $J_{+}$on the state $\psi$ (uud) should give zero. This requires the combination $\uparrow \uparrow \downarrow[\psi(123)+\psi(213)]$ for the first term in Eq. (1).

3. The quark-quark forces are charge independent, and the uud state in Eq. (1) represents a state of isospin $\frac{1}{2}$. This means that applying the type raising operator $d \rightarrow u$ should give zero. Doing so gives

\footnotetext{
${ }^{1}$ This state does not include total orbital angular momentum, which will be considered later in the paper.

${ }^{2}$ The uud representation of the proton wave function has been called the "uds basis" in its application to the Isgur-Karl quark model. See, for instance, Ref. [3] .
} 


$$
\psi(\mathrm{uuu})=N \mathrm{uuu}\{\uparrow \uparrow \downarrow[\phi(123)+\phi(213)]-\uparrow \downarrow \uparrow \phi(123)-\downarrow \uparrow \uparrow \phi(213)\} .
$$

We use the fact that the wave function is completely symmetrized by Eq. (2) to rewrite Eq. (3) as

$$
\psi(\mathrm{uuu})=N \text { uuu } \uparrow \downarrow[\phi(123)+\phi(213)-\phi(132)-\phi(231)] .
$$

For this to equal zero requires the relation

$$
\phi(123)+\phi(213)=\phi(132)+\phi(231)
$$

between different orderings of the variables in the function $\phi$. The three quark wave function is given by Eq. (1) with this constraint on $\phi$.

The wave function for a spin down proton is given by the spin lowering operation $\uparrow \rightarrow \downarrow$ on Eq. (1). The neutron wave function is given by the type lowering operation $u \rightarrow d$ on Eq. (1). This gives, after rewriting the result in the ddu order,

$$
\psi(\mathrm{ddu})=-N \mathrm{ddu}\{\uparrow \uparrow \downarrow[\phi(123)+\phi(213)]-\uparrow \downarrow \uparrow \phi(123)-\downarrow \uparrow \uparrow \phi(213)\} .
$$

As an example of the completely general nature of the wave function in Eq. (1), the mixed symmetry wave function $\mid N_{M}>$ in the Isgur-Karl model [4] is given by Eq. (1) with

$$
\phi(123)=\Phi^{\lambda}(2,3,1),
$$

where $\Phi^{\lambda}(2,3,1)$ is the Isgur-Karl mixed symmetry spatial function that is symmetric in its first two coordinates. If the function $\phi(123)$ in Eq. (1) were completely symmetric in all three coordinates, then Eq. (1) would represent the simplest form of the wave function for the $\mathrm{SU}(6)$ symmetric quark model. Although $\mathrm{SU}(6)$ wave functions have often been given with various linear combinations of the $\mathrm{u}$ and $\mathrm{d}$ quarks and spin states, they all can be rewritten in the form of Eq. (1) $[2,5,6]$.

In order to use the general three body wave function of Eq. (1) for DIS, we integrate the spatial functions over the coordinates of the unstruck spectator quarks. For the general case, this leads to four quark spin distributions:

$$
\begin{aligned}
& u \uparrow\left(x, Q^{2}\right)=2 N^{2}\left[2 \phi(\mathbf{1 2 3})^{2}+\phi(2 \mathbf{1 3})^{2}+2 \phi(\mathbf{1 2 3}) \phi(2 \mathbf{1 3})\right] \\
& u \downarrow\left(x, Q^{2}\right)=2 N^{2} \phi(\mathbf{1 2 3})^{2} \\
& d \uparrow\left(x, Q^{2}\right)=2 N^{2} \phi(123)^{2} \\
& d \downarrow\left(x, Q^{2}\right)=2 N^{2}\left[\phi(123)^{2}+\phi(123) \phi(213)\right] .
\end{aligned}
$$

In each case, the coordinate in boldface represents the struck quark, and the other two coordinates are integrated over. The $x$ variable in the quark distribution is that of the struck quark. The dependence of the quark distributions on $Q^{2}$, the four-momentum transfer to the nucleon squared, follows from the mechanics of deep inelastic scattering. We will drop the $Q^{2}$ variable from the notation, but its presence should be kept in mind. Our general analysis applies for any $Q^{2}$ large enough to eliminate interference between two different 
struck quarks. Because we are only interested here in the high $x$ region, we do not include sea quarks or explicit gluonic effects which are expected to be small for $x>0.3$.

The structure functions for unpolarized DIS are given by

$$
\begin{aligned}
& F_{1 p}(x)=\frac{1}{9}[4 u(x)+d(x)] \\
& F_{1 n}(x)=\frac{1}{9}[u(x)+4 d(x)] .
\end{aligned}
$$

From this we see that if $u(x)=2 d(x)$, as with $\mathrm{SU}(6)$ symmetry, the ratio $F_{1 n} / F_{1 p}$ would be constant at $\frac{2}{3}$ for all $x$. But the experimental ratio falls with increasing $\mathrm{x}$ above 0.3 , seeming to approach $\frac{1}{4}$ as $\mathrm{x}$ nears 1 [7]. ${ }^{3}$ From Eqs. (12) and (13), we see that this would follow immediately if

$$
d(x) / u(x) \rightarrow 0 \text { as } x \rightarrow 1 .
$$

A crucial question for polarized DIS is what implications a vanishing of the ratio $d(x) / u(x)$ would have for polarized structure functions as $x \rightarrow 1$. From Eqs. (8)-(11), we see that $d(x) / u(x)$ will vanish if the first quark in $\phi(123)$ dominates as $x \rightarrow 1$. Then the dominant quark in a proton as $x \rightarrow 1$ will be the u quark with the same spin as the proton. The result of this is that the spin dependent structure function $g_{1 p}$ will equal $F_{1 p}$ as $x \rightarrow 1$. The asymmetry parameter $A_{1 p}$ becomes equal to $g_{1 p} / F_{1 p}$ for $x=1$, so $A_{1 p}$ will approach 1 as $x \rightarrow 1$. The neutron structure function ratio $g_{1 n} / F_{1 n}$, and $A_{1 n}$, will also approach 1 as $x \rightarrow 1$. The conclusion that the ratio $F_{1 n} / F_{1 p}$ approaching $\frac{1}{4}$ as $x \rightarrow 1$ implies that $A_{1} \rightarrow 1$ as $x \rightarrow 1$ has been reached previously in several different models [10-12], but our result is model independent and must hold in any three quark model if the Pauli principle holds, and angular momentum and isospin are conserved.

Other ratios of interest for high $x$ are the quark spin distributions $\Delta u / u$ and $\Delta d / d$. The ratio $\Delta u / u$ will approach 1 as $x \rightarrow 1$, because of the dominance of $u \uparrow$, but we have to look in more detail at $\Delta d / d$. From Eqs. (10) and (11) we get the ratio

$$
\frac{\Delta d(x)}{d(x)}=\frac{-\phi(123) \phi(213)}{\left[2 \phi(123)^{2}+\phi(123) \phi(213)\right]} .
$$

This ratio depends on the interference term in the numerator. For SU(6) symmetry, this ratio would be $-\frac{1}{3}$. It will remain negative if $\mathrm{SU}(6)$ breaking is not so radical that $\phi(213)$ changes sign, which is unlikely since that would require a node in the three quark wave function. Thus, our model independent prediction is that $-\frac{1}{3} \leq \Delta d / d \leq 0$ for all $x$, independently of the behavior of the ratio $F_{1 n} / F_{1 p}$. Measurements of $\Delta d / d$ up to $x=0.6$ are within these limits [1].

The bound $-\frac{1}{3} \leq \Delta d / d \leq 0$ for all $x$ is model independent, and should hold for any three quark bound state satisfying the Pauli principle, and conservation of angular momentum

\footnotetext{
${ }^{3}$ There is some ambiguity, due to binding effects, in the extraction of $F_{1 n}$ from the measured deuteron structure function, so some analyses find a somewhat higher limit of $\sim 0.4$ for $F_{1 n} / F_{1 p}$ as $x \rightarrow 1$. See Refs. [8] and [9].
} 
and isospin. There has been one prediction [13] that $\Delta d / d \rightarrow 1$ as $x \rightarrow 1$, which violates this bound. A perturbative QCD argument is made in Ref. [13] that spin up quarks (both the $u \uparrow$ quark and the $d \uparrow$ quark) dominate as $x$ becomes large. While this is possible for the $u \uparrow$ quark, it can not be true for the $d \uparrow$ quark. It follows from Eq. (5) that only the first quark in the wave function (1) can be dominant. Thus, the only way for the wave function to be dominated by a spin up quark is if it is a $u \uparrow$ quark (and not a $d \uparrow$ quark) that dominates. The ratio $\Delta d / d$ is limited to be between $-\frac{1}{3}$ and 0 , and cannot approach 1 for any $x$. We also see from Eqs. (8)-(11) that, if a spin up quark dominates as $x \rightarrow 1$, then both $d \uparrow$ and $d \downarrow$ must be negligible as $x \rightarrow 1$. This means that the perturbative QCD assumption that a spin up quark dominates as $x \rightarrow 1$ should also lead to the prediction that $F_{1 n} / F_{1 p} \rightarrow \frac{1}{4}$.

We have not explicitly included orbital angular momentum, but we do not expect it to affect our analysis as $x \rightarrow 1$. This is because the ratio of perpendicular to longitudinal momentum goes to zero as $x \rightarrow 1$, leading to $L_{z}=0$. The wave function in Eq. (1) is general enough that it includes orbital angular momentum if $L_{z}=0$. Relativistic effects could reduce $A_{1}$ because the small components of the Dirac wave function reduce the quark spin projection. However this effect also depends on the ratio of perpendicular to longitudinal momentum, so that it too does not affect our results for $x \rightarrow 1$.

The quark spin distributions of Eqs. (8)-(11) can be used to get the most general valence quark form, consistent with the Pauli principle, and conservation of angular momentum and isospin, for the ratio $g_{1} / F_{1}$ of spin dependent to unpolarized structure functions for any $x$. The result is

$$
\begin{aligned}
\frac{g_{1 p}}{F_{1 p}} & =\frac{8\left[\phi(\mathbf{1 2 3})^{2}+\phi(\mathbf{1 2 3}) \phi(2 \mathbf{1 3})\right]-\phi(12 \mathbf{3}) \phi(21 \mathbf{3})}{8\left[\phi(\mathbf{1 2 3})^{2}+\phi(2 \mathbf{1 3})^{2}+\phi(\mathbf{1 2 3}) \phi(2 \mathbf{1 3})\right]+2 \phi(12 \mathbf{3})^{2}+\phi(12 \mathbf{3}) \phi(21 \mathbf{3})} \\
\frac{g_{1 n}}{F_{1 n}} & =\frac{2\left[\phi(\mathbf{1 2 3})^{2}+\phi(\mathbf{1 2 3}) \phi(2 \mathbf{1 3})\right]-4 \phi(12 \mathbf{3}) \phi(21 \mathbf{3})}{2\left[\phi(\mathbf{1 2 3})^{2}+\phi(2 \mathbf{1 3})^{2}+\phi(\mathbf{1 2 3}) \phi(2 \mathbf{1 3})\right]+4\left[2 \phi(12 \mathbf{3})^{2}+\phi(12 \mathbf{3}) \phi(21 \mathbf{3})\right]}
\end{aligned}
$$

For SU(6) symmetry, all $\phi$ terms would be equal, so the predicted ratios would be constant, given by $g_{1 p} / F_{1 p}=5 / 9$ and $g_{1 n} / F_{1 n}=0$. SU(6) breaking, with the term $\phi(\mathbf{1 2 3})^{2}$ dominating as $x \rightarrow 1$, as discussed above, leads to each ratio approaching 1 as $x \rightarrow 1$. The quark interference term for the third quark is larger for the neutron ratio. This suggests that the ratio $g_{1} / F_{1}$ should approach the limiting value of 1 more slower for the neutron than for the proton, which has been observed in high $x$ measurements.

The model independent prediction that $A_{1 p}$ and $A_{1 n}$ each approach 1 as $x \rightarrow 1$ (if $\left.F_{1 n} / F_{1 p} \rightarrow \frac{1}{4}\right)$ is a challenge for experiment. Current measurements indicate that $A_{1 p}$ may be increasing above the $\mathrm{SU}(6)$ value of $\frac{5}{9}$, but it is not clear that the trend around $x=0.6$ is steep enough to reach 1 at $x=1 . A_{1 n}$ is positive in the new measurement at $x=0.6$ [1], but is still not close to 1 . Experiments at higher $x$ will be needed to test the $x \rightarrow 1$ limits. The experimental tests are complicated by a number of problems. It is difficult to achieve accurate asymmetry measurements at large $x$. The $Q^{2}$ dependence increases (beyond QCD evolution) as $x \rightarrow 1$. For $A_{1 n}$, unfolding nuclear effects from either deuteron or helium asymmetries becomes more uncertain as $x \rightarrow 1$. An important question is how high an $x$ is high enough to test the $x \rightarrow 1$ predictions.

I would like to thank Zein-Eddine Meziani for useful discussions about DIS. 


\section{REFERENCES}

[1] X. Zheng et. al., Phys. Rev. Lett. 92,(2004) 012004.

[2] J. Franklin, Phys. Rev. 184 (1968) 1807.

[3] S. Capstick and N. Isgur, Phys. Rev. D 34 (1986) 2809.

[4] N. Isgur and G. Karl, Phys. Rev. D 19 (1970) 2653; D23 (1981) 817(E).

[5] W. Rolnick, Phys. Rev. D 25 (1982) 2439, D 26 (1982) 1804(E).

[6] Z. Dziembowski, Phys. Rev. D 37 (1988) 768.

[7] A. Bodek et. al., Phys. Rev. Lett. 30, (1973) 1087; J. S. Poucher et. al., Phys. Rev. Lett. 32 (1974) 118; A. Bodek et. al., Phys. Lett. 51B (1974) 417.

[8] L. W. Whitlow et al., Phys. Lett. B282 (1992) 475.

[9] W. Melnitchouk and A. W. Thomas, Phys. Lett. 377 (1996) 11.

[10] F. E. Close, Phys. Lett. 43B (1973) 422.

[11] J. Kaur, Nucl. Phys. B128 (1977) 219.

[12] N. Isgur, Phys. Rev. D 59 (1999) 034031.

[13] G. R. Farrar and D. R. Jackson, Phys. Rev. Lett. 35 (1975) 1416. 